ARQGA / 944

\title{
CRITÉRIOS HISTOLÓGICOS DE ESOFAGITE NA DOENÇA DO REFLUXO GASTROESOFÁGICO. Reavaliação da sensibilidade da pHmetria esofágica de 24 horas
}

\author{
Rowilson FLORA-FILHO*, Luiz Heraldo CÂMARA-LOPES** e Bruno ZILBERSTEIN***
}

RESUMO - Os critérios de esofagite histológica pouco se modificaram desde os achados de Ismail-Beiji, Pope (1970) e Weinstein (1975). O diagnóstico da doença do refluxo gastroesofágico modificou-se com o advento da pHmetria esofágica ambulatorial de 24 horas que foi proposta como um método de elevada sensibilidade. Neste estudo selecionaram-se 35 pacientes com esofagite histológica que foram, na seqüencia, submetidos a pHmetria esofágica ambulatorial de 24 horas. Determinaram-se diferenças dos achados histológicos de acordo com padrão de refluxo, graus de esofagite endoscópica e grupos etários. Concluiu-se que a sensibilidade da pHmetria esofágica ambulatorial de 24 horas foi de $60.0 \%$, notandose tendência a maiores alterações teciduais em pacientes com padrões mais severos de refluxo (supino e combinado). Demonstrou-se que nas esofagites endoscópicas mais severas, ocorreu maior exocitose de células inflamatórias no epitélio esofágico. Não houve diferenças de achados histológicos em pacientes com doença do refluxo gastroesofágico conforme grupos etários.

DESCRITORES - Esofagite. Refluxo gastroesofágico. Manometria.

\section{INTRODUÇÃO}

Os aspectos microscópicos do esôfago distal na doença do refluxo gastroesofágico (DRGE) foram inicialmente determinados em 1955. Através de exame de peças de autopsia, consideraram-se sinais histológicos de esofagite a presença de soluções de continuidade ou infiltração inflamatória da lâmina própria ${ }^{(9,12)}$.
Na década de 60, com o advento da esofagoscopia, tornouse possível o diagnóstico precoce da DRGE ${ }^{(15)}$.

ISMAIL-BEIJI et al. ${ }^{(8)}$, através de biopsias endoscópicas por sucção colhidas com auxílio de manometria, determinaram os critérios histológicos de esofagite por refluxo gastroesofágico. Estes critérios são, ainda hoje, os mais utilizados: (a) espessamento da camada basal $>15 \%$ em relação à espessura

\footnotetext{
* Pós-Graduando do Departamento de Gastroenterologia da Faculdade de Medicina da Universidade de São Paulo, São Paulo, SP.

** Laboratório de Patologia Cirúrgica e Molecular. Hospital Sírio-Libanês, São Paulo, SP.

*** Professor-Associado do Departamento de Gastroenterologia da Faculdade de Medicina da Universidade de São Paulo.

Endereço para correspondência: Dr. Bruno Zilberstein - Avenida Brasil, 703 - 01431-000 - São Paulo, SP.
} 
total do epitélio; (b) extensão das papilas até $2 / 3$ da camada epitelial total; (c) presença de neutrófilos na lâmina própria ${ }^{(8)}$.

O estudo de fragmentos de biopsia que não incluíam a lâmina própria ou continham pouco tecido, levou ao estudo de critérios adicionais: a dilatação de vênulas (congestão ou ectasia) com extravasamento de hemácias no interior das papilas e a infiltração de células inflamatórias no epitélio (exocitose) ${ }^{(7)}$.

Controvérsias sobre estes critérios surgiram com os achados de WEINSTEIN et al. ${ }^{(17)}$, que realizaram biopsias por sucção em pacientes assintomáticos para refluxo gastroesofágico: $55 \%$ das biopsias advindas dos $2,5 \mathrm{~cm}$ distais e $19 \%$ das provenientes acima de $2,5 \mathrm{~cm}$ do esfíncter inferior do esôfago, exibiam algumas características histológicas segundo os critérios de ISMAIL-BEIJI et al. ${ }^{(8)}$. Para dirimir controvérsias, propuseram que os achados histológicos abaixo de 2,5 cm distais esofágicos seriam conseqüência do "refluxo fisiológico não-patológico"(17).

Porém a maioria destes critérios foi proposta antes da aplicação clínica de importantes ferramentas para o diagnóstico da DRGE como a pHmetria esofágica ambulatorial de 24 horas (pHM-24h). Esta tem sido proposta como método de alta especificidade e alta sensibilida$\mathrm{de}^{(3,4)}$. Através da pHM-24h, determinam-se três padrões de refluxo nos portadores de DRGE: ortostático, supino e combinado. Uma das importâncias destes diferentes padrões está relacionada com a gravidade da esofagite de refluxo. Foi demonstrado que o padrão combinado, seguido do supino leva a mais complicações do que o padrão ortostático ${ }^{(6)}$.

O objetivo principal deste trabalho é reavaliar a sensibilidade da pHM-24h em pacientes selecionados a partir de achados histológicos compatíveis com esofagite, determinados segundo critérios acima descritos.

Secundariamente procurou-se determinar se existem diferenças entre os padrões de refluxo gastroesofágico determinados pela $\mathrm{pHM}$ $24 \mathrm{~h}$ e os achados microscópicos do esôfago distal em pacientes portadores de esofagite histológica.

Procurou-se, também, especificar quais dos critérios histológicos acima descritos relacionam-se mais diretamente à esofagoscopia e se há alguma diferença de prevalência em relação a grupos etários.

\section{PACIENTES E MÉTODOS}

\section{Pacientes}

Foram selecionados prospectivamente 50 pacientes seqüencialmente encaminhados ao Serviço de Endoscopia Digestiva Alta da Santa Casa de Poços de Caldas, Poços de Caldas, MG, com sintomas (típicos ou atípicos) compatíveis com DRGE. Após a realização de endoscopia digestiva alta (EDA), determinou-se o diagnóstico endoscópico e em seguida foram colhidos fragmentos de biopsia endoscópica $2,5 \mathrm{~cm}$ acima da implantação da linha zeta (transição esofagogástrica). Foram excluídos pacientes portadores de esôfago de Barrett e em vigência de tratamento com bloqueadores $\mathrm{H}_{2}$ ou bloqueadores de bomba de prótons, incluindose aqueles sem medicação há mais de 15 dias. Após exame histológico dos fragmentos, selecionou-se 40 pacientes que satisfizeram os critérios de inclusão e que apresentaram pelo menos um dos critérios histológicos de esofagite. Dos 40 pacientes aos quais foi proposta a realização de pHM-24h, 35 pacientes aceitaram $(n=35)$.

\section{Métodos}

\section{Endoscopia digestiva alta}

Todos pacientes foram submetidos a videoendoscopia digestiva alta com padronização já descrita ${ }^{(5,6)}$. A esofagite endoscópica foi classificada de grau 0 a 4, conforme achados de esôfago distal: grau 0 - ausência de soluções de continuidade (incluindo, além de aspecto normal, presença de eritema, edema, friabilidade ou palidez); grau Ierosões planas isoladas ou múltiplas, não confluentes; grau II - erosões planas confluentes sem atingir toda circunferência esofágica; grau III - erosões confluentes atingindo toda circunferência esofágica; grau IV - presença de complicações como ulcerações ou reduções de calibre esofágico. No grupo selecionado, não havia pacientes portadores de esôfago de Barrett.

$\mathrm{Na}$ retirada do aparelho foram colhidos pelo menos cinco fragmentos de mucosa do esôfago distal nos quadrantes circunferenciais, pelo menos $2,5 \mathrm{~cm}$ acima da transição esofagogástrica endoscopicamente determinada. Os fragmentos foram acondicionados em frascos com solução de formalina tamponada e enviados para estudo histológico entre 24 a 48 horas após a colheita.

\section{Estudo histológico}

Os fragmentos do esôfago distal, corados com hematoxilina-eosina, foram examinados por um dos patologistas (LHCL), além de um autor (RFF).

Classificou-se a presença dos seguintes parâmetros:

a. alongamento de papilas: extensão até $2 / 3$ da camada epitelial total;

b. espessamento da camada basal: presença de células da camada basal atingindo mais do que $15 \%$ da camada epitelial total;

c. exocitose: presença de neutrófilos e/ou eosinófilos e/ou monócitos intraepiteliais;

d. ectasia vascular ou extravasamento: dilatação das vênulas das papilas com congestão ou extravasamento de hemácias para epitélio circunjacente.

O diagnóstico de esofagite histológica foi positivo quando havia pelo menos um dos parâmetros acima citados presentes na lâmina examinada, independente do fragmento encontrado. Para achado positivo foi dado valor numérico de 1 e negativo, 0 . 
Flora-Filho R, Câmara-Lopes LH, Zilberstein B. Critérios histológicos de esofagite na doença do refluxo gastroesofágico. Reavaliação da sensibilidade da pHmetria esofágica de 24 horas

\section{3. pHmetria esofágica ambulatorial de $24 \mathrm{~h}$}

Após localização manométrica do esfíncter do esôfago inferior, procedeu-se à colocação do cateter conforme técnica já descrita ${ }^{(6)}$. Para o cálculo de normalidade foram utilizados os seguintes parâmetros, segundo critérios de SCHINDLBECK et al. ${ }^{(14)}$ : percentagem de tempo total de refluxo ácido até $3,4 \%$, percentagem de tempo de refluxo ácido em posição ortostática até $8,2 \%$, percentagem de tempo de refluxo ácido em posição supina até 3,0\%.

\section{Cálculos e estatística}

A sensibilidade foi definida como capacidade de determinado teste diagnóstico detectar a doença quando realmente presente (relação entre real-positivo/(real-positivo + falso-negativo) sendo expressa em percentagem).

Para o cálculo estatístico da distribuição da mediana dos achados histológicos utilizou-se o teste não paramétrico de Kruskal-Wallis. Considerou-se $P$ como significativo quando menor do que $0,05(P<0,050)$.

\section{RESULTADOS}

A idade dos 35 pacientes variou entre 12 e 71 anos (média de idade de 37,37 anos), sendo 24/35 (68,57\%) do sexo masculino e 11/ $35(31,43 \%)$ do sexo feminino.

O achado histológico mais prevalente foi exocitose (c) em 24/35 $(65,57 \%)$, seguido de alongamento de papilas (a) encontrado em 22/ 35 (62,85\%), ectasia ou extravasamento (d) em 20/35 (57,14\%) e espessamento da camada basal (b) em 18/35 (51,43\%) (Tabela 1).
A Tabela 2 e a Figura 1 mostram os 35 pacientes separados conforme achado da $\mathrm{pHM}-24 \mathrm{~h}$ : grupo I com $\mathrm{pHM}-24 \mathrm{~h}$ normal, grupo II com pHM-24h de padrão ortostático e grupo III, padrão supino e combinado.

FIGURA 1 - Distribuição dos pacientes em grupos estratificados segundo pHmetria ambulatorial de $24 \mathrm{~h}$ e conforme achados histológicos de esofagite $(\mathrm{A}=$ alongamento de papilas; $\mathrm{B}=$ espessamento da camada basal; $\mathrm{C}=$ presença de exocitose; $\mathrm{D}=$ presença de extravasamento ou ectasia)

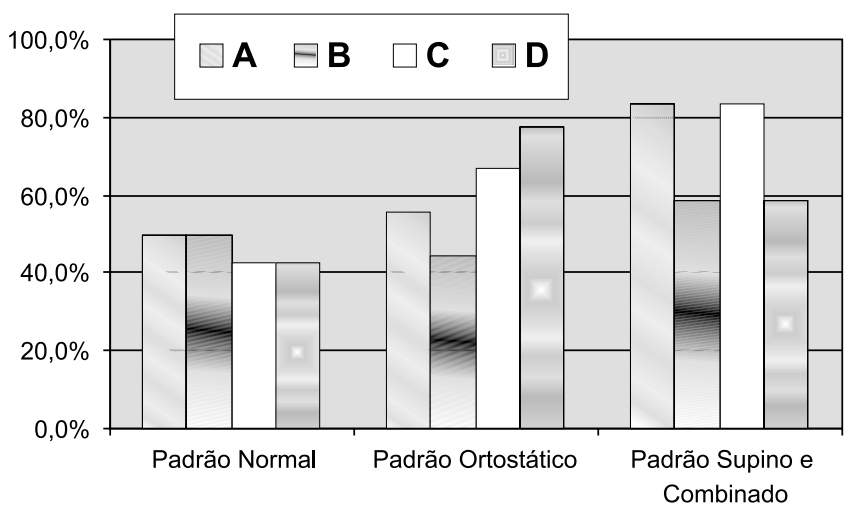

O agrupamento dos pacientes por achados de esofagite endoscópica determinou os grupos (1) com esofagite de refluxo grau 0 (leve), (2) com graus I e II (moderada), e (3) graus III e IV (severa), relatados na Tabela 3 e Figura 2.

TABELA 1 - Distribuição dos achados histológicos dos pacientes selecionados $(\mathrm{n}=35)$

\begin{tabular}{cccccccc}
\hline \multicolumn{2}{c}{ Alongamento de papilas } & \multicolumn{2}{c}{ Espessamento camada basal } & \multicolumn{2}{c}{ Presença de exocitose } & \multicolumn{2}{c}{ Extravasamento ou ectasia } \\
\hline $22 / 35$ & $62,85 \%$ & $18 / 35$ & $51,43 \%$ & $24 / 35$ & $65,57 \%$ & $20 / 35$ & $57,14 \%$ \\
\hline
\end{tabular}

TABELA 2 - Distribuição das percentagens relativas dos achados histológicos em pacientes estratificados segundo padrão da pHmetria ambulatorial de 24h (grupo 1: padrão normal, grupo 2: padrão ortostático e grupo 3: padrão supino e combinado

\begin{tabular}{|c|c|c|c|c|c|c|c|c|c|}
\hline & \multirow[b]{2}{*}{$\mathbf{N}$} & \multicolumn{2}{|c|}{$\begin{array}{l}\text { Alongamento } \\
\text { de papilas }\end{array}$} & \multicolumn{2}{|c|}{$\begin{array}{c}\text { Espessamento } \\
\text { de camada } \\
\text { basal }\end{array}$} & \multicolumn{2}{|c|}{ Exocitose } & \multicolumn{2}{|c|}{$\begin{array}{c}\text { Extravasamento } \\
\text { ou ectasia }\end{array}$} \\
\hline & & $\mathbf{N}$ & $\%$ & $\mathbf{N}$ & $\%$ & $\mathbf{N}$ & $\%$ & $\mathbf{N}$ & $\%$ \\
\hline Padrão normal & $14 / 35$ & $7 / 14$ & 50,0 & $7 / 14$ & 50,0 & $6 / 14$ & 42,9 & $6 / 14$ & 42,9 \\
\hline Padrão ortostático & $9 / 35$ & $5 / 9$ & 55,6 & $4 / 9$ & 44,4 & $6 / 9$ & 66,7 & $7 / 9$ & 77,8 \\
\hline \multirow[t]{2}{*}{ Padrão supino e combinado } & $12 / 35$ & $10 / 12$ & 83,3 & $7 / 12$ & 58,3 & $10 / 12$ & 83,3 & $6 / 12$ & 58,3 \\
\hline & & \multicolumn{2}{|c|}{$P=0,196$} & \multicolumn{2}{|c|}{$P=0,820$} & \multicolumn{2}{|c|}{$P=0,107$} & \multicolumn{2}{|c|}{$P=0,265$} \\
\hline
\end{tabular}


TABELA 3 - Distribuição das percentagens relativas dos achados histológicos em pacientes estratificados segundo achado endoscópico (Grupo 1: esofagite grau 0 (leve), grupo 2: esofagite grau I e II (moderada) e grupo 3: esofagite grau III e IV (severa))

\begin{tabular}{|c|c|c|c|c|c|c|c|c|c|}
\hline & \multirow[b]{2}{*}{$\mathbf{N}$} & \multicolumn{2}{|c|}{$\begin{array}{l}\text { Alongamento } \\
\text { de papilas }\end{array}$} & \multicolumn{2}{|c|}{$\begin{array}{c}\text { Espessamento } \\
\text { de camada } \\
\text { basal }\end{array}$} & \multicolumn{2}{|c|}{ Exocitose } & \multicolumn{2}{|c|}{$\begin{array}{c}\text { Extravasamento } \\
\text { ou ectasia }\end{array}$} \\
\hline & & $\mathbf{N}$ & $\%$ & $\mathbf{N}$ & $\%$ & $\mathbf{N}$ & $\%$ & $\mathbf{N}$ & $\%$ \\
\hline Esofagite grau 0 (leve) & $12 / 35$ & $6 / 12$ & 50,0 & $5 / 12$ & 41,7 & $6 / 12$ & 50,0 & $5 / 12$ & 41,7 \\
\hline Esofagite grau I e II (moderada) & $15 / 35$ & $11 / 15$ & 73.3 & $8 / 15$ & 53.3 & $8 / 15$ & 53.3 & $9 / 15$ & 60.0 \\
\hline \multirow[t]{2}{*}{ Esofagite grau III e IV (severa) } & $8 / 35$ & $5 / 8$ & 62.5 & $5 / 8$ & 62.5 & $8 / 8$ & 100.0 & $6 / 8$ & 75.0 \\
\hline & & \multicolumn{2}{|c|}{$P=0,469$} & \multicolumn{2}{|c|}{$P=0,655$} & \multicolumn{2}{|c|}{$* * P=0,050$} & \multicolumn{2}{|c|}{$P=0,333$} \\
\hline
\end{tabular}

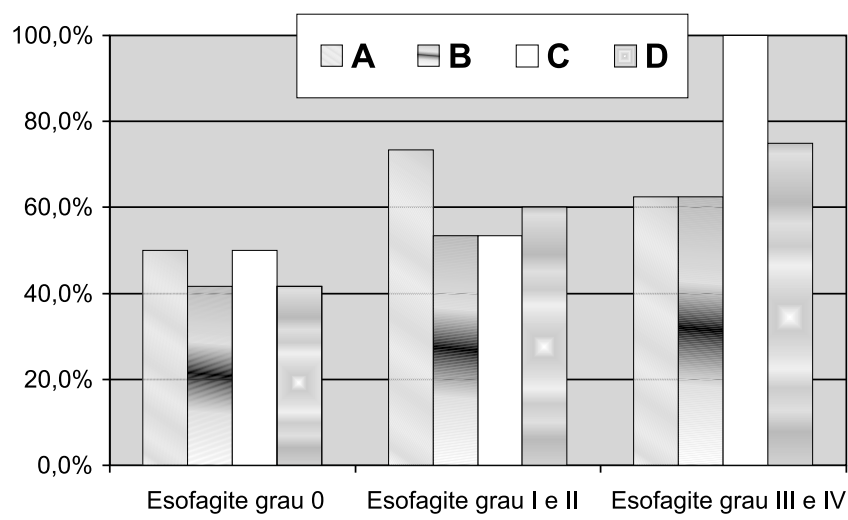

FIGURA 2 - Distribuição dos pacientes em grupos estratificados segundo achado endoscópico e conforme achados histológicos de esofagite $(\mathrm{A}=$ alongamento de papilas; $\mathrm{B}=$ espessamento da camada basal; $\mathrm{C}=$ presença de exocitose; $\mathrm{D}=$ presença de extravasamento ou ectasia) $* * P=0,050$ (significativo) na coluna $\mathrm{C}$.

Quando os pacientes foram estratificados por grupo etário, grupo I com idade menor ou igual a 25 anos, grupo II com idade maior do que 25 e menor ou igual a 40 anos e grupo III com idade maior do que 40 anos, observam-se os achados demonstrados na Tabela 4 e Figura 3.

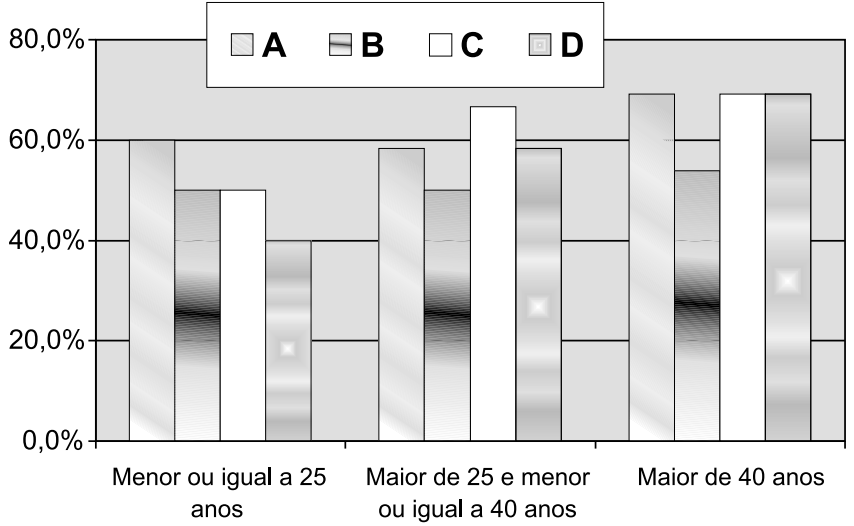

FIGURA 3 - Distribuição dos pacientes em grupos estratificados segundo idade cronológica e conforme achados histológicos de esofagite $(\mathrm{A}=$ alongamento de papilas; $\mathrm{B}=$ espessamento da camada basal; $\mathrm{C}=$ presença de exocitose; $\mathrm{D}=$ presença de extravasamento ou ectasia)

O cálculo da sensibilidade da $\mathrm{pHM}-24 \mathrm{~h}$ no total de pacientes $(\mathrm{n}=$ 35 ) portadores de esofagite histológica foi de 60,0\%. Quanto à EDA, a sensibilidade foi de $65,7 \%$.

TABELA 4 - Distribuição das percentagens relativas dos achados histológicos em pacientes estratificados segundo idade cronológica (Grupo I menor ou igual a 25 anos, grupo II maior de 25 e menor ou igual a 40 anos e grupo III maior de 40 anos)

\begin{tabular}{|c|c|c|c|c|c|c|c|c|c|}
\hline & \multicolumn{3}{|c|}{$\begin{array}{l}\text { Alongamento } \\
\text { de papilas }\end{array}$} & \multicolumn{2}{|c|}{$\begin{array}{l}\text { Espessamento } \\
\text { de camada } \\
\text { basal }\end{array}$} & \multicolumn{2}{|c|}{ Exocitose } & \multicolumn{2}{|c|}{$\begin{array}{c}\text { Extravasamento } \\
\text { ou ectasia }\end{array}$} \\
\hline & $\mathbf{N}$ & $\mathbf{N}$ & $\%$ & $\mathbf{N}$ & $\%$ & $\mathbf{N}$ & $\%$ & $\mathbf{N}$ & $\%$ \\
\hline Menor ou igual a 25 anos & $10 / 35$ & $6 / 10$ & 60,0 & $5 / 10$ & 50,0 & $5 / 10$ & 50,0 & $4 / 10$ & 40,0 \\
\hline Maior de 25 e menor ou igual a 40 anos & $12 / 35$ & $7 / 12$ & 58,3 & $6 / 12$ & 50,0 & $8 / 12$ & 66,6 & $7 / 12$ & 58,3 \\
\hline \multirow[t]{2}{*}{ Maior de 40 anos } & $13 / 35$ & $9 / 13$ & 69,2 & $7 / 13$ & 53,8 & $9 / 13$ & 69,2 & $9 / 13$ & 69,2 \\
\hline & & \multicolumn{2}{|c|}{$P=0,845$} & \multicolumn{2}{|c|}{$P=0,977$} & \multicolumn{2}{|c|}{$P=0,684$} & \multicolumn{2}{|c|}{$P=0,382$} \\
\hline
\end{tabular}




\section{DISCUSSÃO}

Embora os vários critérios de diagnóstico (clínicos, endoscópicos, funcionais) da DRGE sejam muito dinâmicos, ainda persiste o consenso de que a esofagite histológica, juntamente com outros critérios ou mesmo isoladamente, é o pilar para o diagnóstico de certeza da DRGE. Após os estudos de ISMAIL-BEIJI et al. ${ }^{(8)}$, sedimentados pelos achados de WEINSTEIN et al. ${ }^{(17)}$ quanto à padronização do local de colheita dos fragmentos esofágicos, poucas mudanças se seguiram nos achados da histologia do esôfago distal em pacientes portadores de DRGE. Destacam-se critérios histológicos de esofagite em pediatria, como o achado de eosinófilos intra-epiteliais ${ }^{(18)}$, que foram parcialmente reproduzidos em adultos, mostrando ser um critério de baixa sensibilidade em casos de DRGE sem soluções de continuidade ${ }^{(2)}$.

Com o advento de novas técnicas diagnósticas, como a pHM24h, descortinou-se a possibilidade de subclassificações da DRGE. Através da pHM-24h caracterizaram-se objetivamente três padrões de refluxo (ortostático, supino e combinado) ${ }^{(6)}$ diferentes, com cinéticas aparentemente próprias. Embora esta linha de raciocínio seja tentadora, a volatilidade de conceitos já caracterizou novos padrões de refluxo como o refluxo fisiológico sintomático, também chamado esôfago hipersensível ${ }^{(10,13)}$. A situação atual mostra que a pHM-24h, embora um bom método, não é o final para o diagnóstico da DRGE.

A EDA, há muito sedimentada no diagnóstico da DRGE, foi demonstrada como de sensibilidade intermediária com controvérsias nos casos incipientes ${ }^{(5)}$.

$\mathrm{O}$ estudo do epitélio esofágico em pacientes com DRGE provavelmente se encaminhará, em futuro próximo, para o nível molecular. Diferenças sutis de expressão protéica poderão classificar com mais sensibilidade a intensidade de lesão celular.

A pHM-24h tem sido proposta como método diagnóstico com alta sensibilidade (acima de $90 \%)^{(3,4)}$. Entretanto não é o que se tem visto na prática clínica. Avolumam-se relatos de pacientes com sintomas tido atípicos da DRGE com EDA e pHM-24h normais ${ }^{(1,}$ ${ }^{16)}$ que respondem a tratamento clínico padrão para DRGE. Portanto, achamos oportuna a reavaliação da sensibilidade destes métodos diagnósticos. Nosso achado de sensibilidade de 65,7\% da EDA está dentro da média da literatura ${ }^{(10)}$; entretanto a sensibilidade de $60.0 \%$ para $\mathrm{pHM}-24 \mathrm{~h}$ discorda de grande número de relatos. Salienta-se que nossos dados estão em concordância com outros de nosso meio ${ }^{(11)}$.

Os achados histológicos (Tabela 2 e Figura 1) em relação aos padrões de refluxo mostram que formas mais graves de refluxo (supino e combinado) tendem a maiores alterações histológicas. Este achado é bastante coerente com o aumento de complicações nos padrões de refluxo combinado e supino, em relação ao ortostático ${ }^{(6)}$.

A estratificação dos critérios histológicos pelos graus de esofagite (Tabela 3 e Figura 2) evidencia dados interessantes: o alongamento de papilas e espessamento da camada basal é uniforme nos três grupos. Este achado é bastante compatível com as características de cronicidade da DRGE em que a maioria dos pacientes se situa nas formas moderadas de esofagite endoscópica, ou seja, os tecidos vão desenvolvendo mecanismos de adaptação como maior taxa de "turn-over" celular evidenciada pelo espessamento da camada basal e aumento do fluxo de nutrientes às células remanescentes (alongamento das papilas). Entretanto, nos graus severos de esofagite endoscópica (III e IV) observa-se aumento significativo da exocitose sugerindo ruptura dos mecanismos de defesa dinâmicos com o extravasamento de células inflamatórias para o epitélio.

Como a DRGE é considerada uma doença crônica que se inicia durante idades jovens e com pouca chance de remissão espontânea ${ }^{(5)}$, cabe a pergunta: seriam os mecanismos de defesa epitelial esofágica diferentes durante a idade adulta? A Tabela 4 e a Figura 3 confirmam que não há diferença, achado este de acordo com a observação clínica.

\section{CONCLUSÕES}

No grupo de pacientes estudados, selecionados a partir de achados histológicos de esofagite, a sensibilidade da pHM-24h foi de $60,0 \%$. Não se demonstraram estatisticamente diferenças histológicas entre padrões de refluxo determinados pela pHM-24h; notou-se, porém, tendência a maiores alterações teciduais em pacientes com padrões mais severos de refluxo (supino e combinado). As esofagites endoscópicas mais severas (III e IV) estão associadas com diferença significante de permeação epitelial com aumento de células inflamatórias (exocitose). Apesar da DRGE ter um caráter crônico acompanhando o paciente por anos e sem remissão espontânea, os achados histológicos pouco diferem em relação aos grupos etários (idade adulta).

Flora-Filho R, Câmara-Lopes LH, Zilberstein B. Esophagitis histological criteria and sensitivity reappraisal of the ambulatorial 24-hour pHmetry in gastroesophageal reflux patients. Arq Gastroenterol 2000;37(4):197-202.

ABSTRACT - Little change was observed in the histological criteria of reflux esophagitis since the studies of Ismail-Beiji, Pope (1970) and Weinstein (1975). The 24-hour esophageal pHmetry has been proposed as a high sensitivity method in diagnosis of gastroesophageal reflux disease patients. In this study we selected 35 patients with histological esophagitis and submitted them to 24-hour esophageal pHmetry. We determined histological differences according to reflux pattern, endoscopic esophagitis grades and age. The sensitivity of 24hour esophageal pHmetry was $60.0 \%$ in our patients. There are higher histological alterations in patients with more severe patterns of reflux (supine and combined) and significant difference $(P<0,05)$ in observed quantitative exocytosis between moderate and severe endoscopic esophagitis. There are no difference between histological esophagitis criteria and age groups.

HEADINGS - Esophagitis. Gastroesophageal reflux. Manometry. 


\section{REFERÊNCIAS BIBLIOGRÁFICAS}

1. Armstrong D, Emde C, Inauen W, Blum AL. Diagnostic assessment of gastroesophageal reflux disease: what is possible vs. what is practical? Hepatogastroenterology 1992;39(S-1):3-13.

2. Brown LF, Goldman H, Antonioli DA. Intraepithelial eosinophils in endoscopic biopsies of adults with reflux esophagitis. Am J Surg Pathol 1984;8:809-905.

3. DeMeester TR, Johnson LF, Joseph GJ, Toscano MS, Hall AW, Skinner DB. Patterns of gastroesophageal reflux in health and disease. Ann Surg 1976;184:459-70.

4. DeMeester TR, Wang C-I, Wernly JA, Pellegrini CA, Little AG,Klementschitsch P, Bermudez G, Johnson LF, Skinner DB. Technique, indications, and clinical use of 24-hour esophageal $\mathrm{pH}$ monitoring. J Thorac Cardiovasc Surg 1980;79:656-70.

5. Flora-Filho R, Zilberstein B. Reflux esophagitis and the gastroesophageal reflux disease - cross-sectional study in gastroesophageal reflux disease patients organized by groups. Rev Hosp Clin Fac Med S Paulo 1999;54:61-7.

6. Flora-Filho R, Zilberstein B, Nasi A. Relação entre pHmetria esofagiana ambulatorial de $24 \mathrm{~h}$ e endoscopia digestiva alta em pacientes portadores de esofagite de refluxo. Arq Gastroenterol 1998;35:252-7.

7. Goldman H, Antonioli DA. Mucosal biopsy of the esophagus, stomach, and proximal duodenum. Human Pathol 1982;13:423-48.

8. Ismail-Beigi F, Horton PF, Pope II CE. Histological consequences of gastroesophageal reflux in man. Gastroenterology 1970;58:163-74.

9. Lodge KV. The pathology of non-specific oesophagitis. J Pathol Bacteriol 1955;69:17-24.
10. Nasi A. Doença do refluxo gastroesofágico: reavaliação clínica, endoscópica e da monitorização do pH intraluminar esofágico. São Paulo, 1996. (Tese Doutorado - Faculdade de Medicina da Universidade de São Paulo).

11. Navarro-Rodriguez T, Moraes-Filho JPP, Arakaki E, Chinzon D, Zaterka S, Iriya $\mathrm{K}$, Laudanna AA. The screening sensitivity of endoscopy, acid perfusion test and 24-hour $\mathrm{pH}$-monitoring to evaluate esophagitis in patients with heartburn and histological esophagitis. Arq Gastroenterol 1997;34:148-56.

12. Peters PM. The pathology of severe digestion oesophagitis. Thorax 1955;10:269-86

13. Saraswat VA, Dhiman RK, Mishra A, Naik SR. Correlation of 24-hr esophageal patterns with clinical features and endoscopy in gastroesophageal reflux disease. Dig Dis Sci 1994;39:199-205.

14. Schindlbeck NE, Ippisch H, Klauser AG, Müller-Lissner SA. Which pH threshold is best in esophageal $\mathrm{pH}$ monitoring? Am J Gastroenterol 1991;86:1138-41.

15. Svodoba AC, Knauer CM, Gamble CN, Sommers SC, Monroe LS. Problem in the early diagnosis of peptic esophagitis. Gastrointest Endosc 1967;13:14-7.

16. Waring JP, Lacayo L, Hunter J, Katz E, Suwak B. Chronic cough and hoarseness in patients with severe gastroesophageal reflux disease. Diagnosis and response to therapy. Dig Dis Sci 1995;40:1093-7.

17. Weinstein WM, Bogoch ER, Bowes KL. The normal human esophageal mucosa: a histological reappraisal. Gastroenterology 1975;68:40-4.

18. Winter HS, Madara JL, Stafford RJ, Grand RJ, Quinlan JE, Goldman H Intraepithelial eosinophils: a new diagnostic criterion for reflux esophagitis. Gastroenterology 1982;83:818-23.

Recebido para publicação em 7/12/1999. Aprovado para publicação em 14/3/2000 